ophtalmoplegia is provoked by intra-axial lesions within the higher part of the cerebral trunk, above mesencephalic oculomotor centres; however, a supra-nuclear lesion during pSS has never been reported so far.

Through a literature review, we discuss relevance, aetiology and treatment of CNS involvement in pSS. Data are imprecise and even contradictory. Frequency of CNS involvement in pSS could vary from $0 \%$ to $25 \%$ and might display various patterns: focal or multi focal lesions entailing encephalon, cerebral trunk, cerebellum or medulla, but also more diffuse processes as an aseptic meningo-encephalitis or as a cognitive impairment.

It's pathogenesis is poorly understood. Two mechanisms have been proposed: the first being of an ischaemic nature resulting from a vasculitis of small brain vessels, the second through an autoimmune response (humoral by anti-neuronal antibodies or cytotoxic).

There is no clear consensus concerning the treatment of neurological complications of pSS. Corticosteroïds are usually initiated first; immuno-suppressive agents may be necessary in the absence of improvement.

Conclusion A supranuclear ophtalmoplegia can be observed in pSS; it reinforces the thesis of a CNS involvement in this connective disease, in so far such a characteristic symptom cannot be explained by a Steele-Richarson disease, a multiple sclerosis or vascular lesions. Be aware of CNS involvement in pSS would leed to realise a minor salivary gland biopsy that is the only exam useful to establish the diagnosis.

\section{AB0119 PRIMARY SJOGREN'S SYNDROME ASSOCIATED WITH ANTERIOR HORN CELL DISORDER}

JC Tseng, HH Cheng, RJ Hu, LY Lu. Allergy, Immunology and Rheumatology, Veterans General Hospital, Kaohsiung, Taiwan, R.O.C

\subsection{6/annrheumdis-2001.302}

Background Central nervous system (CNS) involvement in primary Sjogren's syndrome (pSS) is controversial with regard to incidence, aetiology and pathogenesis. In the literature, the incidence of CNS-SS varies widely, ranging from rare to incidence rates of $20 \%$ to $25 \%$. The clinical picture is diverse, ranging from mild cognitive symptoms to fatal cerebrovascular accidents. Objectives We describe a patient with pSS presenting with dysphagia and progressive respiratory failure resulting from anterior horn cell disorder. To the best of our knowledge this is the first case reported in medical literature. The disease responded to corticosteroids and hydroxychloroquine treatment.

Methods A 58-year-old woman had a one year history of dry eyes and dry mouth. She experienced progressive dysphagia and dyspnea ten days prior to admission. She developed progressive respiratory muscle weakness which resulted in respiratory failure and received mechanical ventilation during admission. Examination revealed symmetrically decreased muscle power without sensory impairment and positive Schirmer test. Blood investigations revealed positive ANA, anti-SS-A and elevated IgG level up to $2820 \mathrm{mg} / \mathrm{dl}$. Full blood cell count and creatine kinase level were normal. Sjogren's syndrome was confirmed by minor salivary gland biopsy. Electromyography, nerve conduction velocity and evoked potential studies showed anterior horn cell disorder. MRI of brain revealed negative finding. The disease improved with administration of corticosteroids and hydroxychloroquine. Results
Conclusion Sjoren's syndrome should be considered in the differential diagnosis of patients with motor neuron diseases. Promop diagnosis and treatment are required to prevent further morbidity and mortality.

\section{REFERENCES}

1 Cox PD, Hales RE. CNS Sjogren's syndrome: an underrecognized and underappreciated neuropsychiatric disorder. J Neuropsychiatry Clin Neurosci. 1999;11 (2):241-7

2 Wright RA, O'Duffy JD, Rodriguez M. Improvement of myelopathy in Sjogren's syndrome with chlorambucil and prednisolone therapy. Neurology 1999;52 (2):386-8

3 Ioannidis JP, Moutsopoulos HM. Sjogren's syndrome: too many associations, too limited evidence. The enigmatic example of CNS involvement. Semin Arthritis Rheum. 1999;29(1):1-3

4 Niemela RK, Hakala M. Primary Sjogren's syndrome with severe central nervous system disease. Semin Arthritis Rheum. 1999;29(1):4-13

\section{AB0120 PRIMARY SJOGREN'S SYNDROME ASSOCIAED WITH ANTERIOR HORN CELL DISORDER}

JC Tseng, HH Cheng, RJ Hu, LY Lu. Allergy, Immunology and Rheumatology, Veterans General Hospital, Kaohsiung, Taiwan, R.O.C

\subsection{6/annrheumdis-2001.303}

Background Central nervous system (CNS) involvement in primary Sjogren's syndrome (pSS) is controversial with regard to incidence, aetiology and pathogenesis. In the literature, the incidence of CNS-SS varies widely, ranging from rare to incidence rates of $20 \%$ to $25 \%$. The clinical picture is diverse, ranging from mild cognitive symptoms to fatal cerebrovascular accidents. Objectives We describe a patient with pSS presenting with dysphagia and progressive respiratory failure resulting from anterior horn cell disorder. The disease responded to corticosteroids, hydroychloroquine and azathioprine treatment. To our knowledge this is the first case reported in the medical literature.

Methods A 58-year-old woman had a one year history of dry eyes and dry mouth. She experienced progressive dysphagia and dyspnea ten days prior to admission. She developed progressive respiratory muscle weakness which resulted in respiratory failure and received mechanical ventilation during admission. Examination revealed symmetrically decreased muscle power without sensory impairment. Schirmer test was positive. Blood investigations revealed positive ANA, anti-SS-A and elevated IgG level up to $2820 \mathrm{mg} / \mathrm{dl}$. Full blood cell count and creatine kinase level were normal. Sjogren's syndrome was proved by minor salivary gland biopsy. Electromyography, nerve conduction velocity and evoked potentials studies showed anterior horn cell disorder. MRI of brain revealed negative finding. The disease improved with administration of corticosteroids, hydroxychloroquine and azathioprine.

Results

Conclusion Sjogren's syndrome should be considered in the differential diagnosis of patients with motor neuron diseases. Prompt diagnosis and treatment prevent further morbidity and mortality.

\section{REFERENCES}

1 Cox PD, Hales RE. CNS Sjogren's syndrome: an underrecognized and underappreciated neuropsychiatric disorder. J Neuropsychiatry Clin Neurosci. 1999;11 (2):241-7

2 Wright RA, O'Duffy JD, Rodriguez M. Improvement of myelopathy in Sjogren's syndrome with chlorambucil and prednisolone therapy. Neurology 1999;52 (2):386-8

3 loannidis JP, Moutsopoulos HM. Sjogren's syndrome: too many associations, too limited evidence. The enigmatic example of CNS involvement. Semin Arthritis Rheum. 1999;29(1):1-3 